\title{
Study of the rheological properties and crystallization behavior of branched PP/silicate composites
}

\author{
Kyung Hwa Yoon ${ }^{1}$, Sung Park ${ }^{2}$ and Youn Cheol Kim ${ }^{1}$ \\ Long-chain branched-polypropylene (LCB-PP) was prepared by melt grafting, and LCB-PP/silicate composites were prepared \\ by adding $1-7$ wt $\%$ silicate using a mini-compounder at $190^{\circ} \mathrm{C}$. The chemical structure of the LCB-PP was confirmed by the \\ existence of a $-\mathrm{C}=\mathrm{C}-\mathrm{H}$-stretching peak at $3100 \mathrm{~cm}^{-1}$ in the Fourier transform infrared spectrum. LCB-PP and the LCB-PP/ \\ silicate composites exhibited unusual rheological properties, including high shear-thinning tendency and elasticity. Shear- \\ thinning tendency and elasticity were highest in the composite containing $5 \mathrm{wt} \%$ silicate. These effects were confirmed by \\ oscillatory rheological measurements. The crystallization behavior of LCB-PP and the silicate composite were investigated using \\ a non-isothermal process proposed by Ozawa. The exothermic pattern of the composite is narrower and sharper than those of \\ PP and branched PP. The extending exponents were 3.6 for PP, 2.4 for LCB-PP and 1.5 for the composite. These behaviors \\ can be interpreted by speculating that the silicate in the PP matrix functions as a seed for crystallization and alters the \\ crystallization process.
}

Polymer Journal (2012) 44, 1098-1104; doi:10.1038/pj.2012.68; published online 25 April 2012

Keywords: crystallization; long-chain branch; polypropylene; rheological property; silicate composite

\section{INTRODUCTION}

Polypropylene (PP) has been the fastest growing commodity resin owing to its desirable and beneficial physical properties such as low weight, recyclability and chemical resistance. However, commercial PP exhibits low melt and impact strengths, which limit its use in foaming and automotive applications. Therefore, a great deal of effort has been directed toward enhancing the melt strength and impact strength of PP. ${ }^{1-15}$ To improve its impact strength, impact modifiers have been added to PP, and ethylene propylene rubber has been considered the most effective among them due to its high impact strength over a wide temperature range. These blends, commonly referred to as thermoplastic polyolefin elastomers, have an increasingly important role in the polymer industry, especially in automotive applications.

The melt strength of PP can be improved by various methods such as controlling the molecular weight and distribution, ${ }^{1,2}$ introducing long-chain branches (LCBs), ${ }^{3-11}$ or forming polymer composites with inorganic nanofillers. ${ }^{12-15}$ Among these approaches, the most efficient way to enhance the melt strength of PP is by introducing LCBs within the PP chain. The polymer industry and academic fields have expended much effort to produce branched PPs. Several commercial branched PPs have been created using electron beam irradiation. ${ }^{15}$ However, irradiation causes $\beta$-scission of the PP chains followed by crosslinking and the formation of complex branch structures. Many reports have also described the modification of PP with peroxides in combination with multi-functional monomers. Borsig et al. ${ }^{3}$ reported the possibility of producing branched PP using a solid-state reaction in the presence of furfuryl sulfide (FS) combined with tert-butyl perbenzoate (TBPB). However, the commercial limitations of this method are obvious owing to the use of a polymer-solvent mixture. In response, researchers have studied the melt grafting of PP. Notably, Shuzhao et al. ${ }^{2}$ synthesized branched PP via melt grafting in the presence of glycidyl methacrylate and a novel chain extender.

The crystal growth rate of the long-chain branched-polypropylene (LCB-PP) decreases because the chain transfer from the PP melt to the crystallization interface is restrained by the LCB. Control of the crystal growth rate is crucial for the preparation of LCB-PP because the crystallization kinetics affect the polymer processing. The crystallization behavior of semi-crystalline polymers such as PP is known to be affected by the presence of particulate additives, especially at low concentrations. This feature can affect the overall properties of the nanocomposite; nevertheless, few relevant investigations have been reported. ${ }^{16-19}$ Non-isothermal crystallization studies on semi-crystalline polymers have been widely carried out because the crystallization conditions are analogous to those of real processing. Although an analysis of the non-isothermal crystallization process may be much more complicated than that of isothermal processes, non-isothermal crystallization measurements can provide ample information regarding the crystalline transition. The non-isothermal

${ }^{1}$ Major in Polymer Science and Engineering, Kongju National University, Cheonan, Republic of Korea and ${ }^{2}$ Department of Materials Science \& Engineering, Myongji University, Kyeonggi, Republic of Korea

Correspondence: Professor YC Kim, Major in Polymer Science and Engineering, Kongju National University, Budae-Dong 275, Cheonan, 330-717, Republic of Korea. E-mail: younkim@kongju.ac.kr

Received 22 October 2011; revised 13 February 2012; accepted 4 March 2012; published online 25 April 2012 
kinetics of the process of nucleation and its growth were derived by extending Avrami's equation as proposed by Ozawa, ${ }^{20}$

$$
1-C(T)=\exp \left[\frac{\chi_{\mathrm{C}}(T)}{a^{n}}\right]
$$

in which $n$ depends on the dimension of the growth, and its values range from 2 to $4 ; \chi_{C}(T)$ is the cooling function; and $C(T)$ is the conversion at temperature $T$.

As the microstructures of the silicate and the LCB-PP crystallite may have remarkable effects on the physical properties of the LCB$\mathrm{PP} /$ silicate composites, it is meaningful to study the influence of the silicate on the crystallization process of the branched matrix. Accordingly, a study of the crystallization behavior of the LCB-PP/ silicate composite is a topic of interest.

The LCB-PP melts possess unusual viscoelastic properties such as non-terminal dynamic moduli at low frequency and a high shearthinning tendency. ${ }^{21-27}$ The rheology of the polymer nanocomposites is not affected by the chemical nature of the PP, but rather, by the mesoscopic structure of the hybrid. The clay tactoids form a percolating network because of physical jamming, which offers considerable resistance to deformation, and thus, solid-like behavior. ${ }^{28}$ Large-amplitude dynamic data revealed a decrease in the storage modulus, and a flow-induced alignment of the clay was postulated to occur at a higher strain. A flow-induced alignment is known to alter the rheological properties in the molten state.

This study was carried out to determine the effects of LCB and loading silicate on the rheological properties and the non-isothermal crystallization kinetics of block PP. LCB-PP was prepared by melt grafting in the presence of FS and TBPB. LCB-PP/silicate composites were created using a micro-compounder, and a systematic study was conducted on their rheological properties and non-isothermal crystallization kinetics.

\section{EXPERIMENTAL}

\section{Materials}

Grade BJ110 Block-PP without an anti-oxidant was supplied by a Samsung Total (Daesan, Korea). 'Block PP' indicates an iso-PP and ethylenepropylene rubber blend with a melt index for the PP of $1.0 \mathrm{~g}$ for $10 \mathrm{~min}\left(230^{\circ} \mathrm{C}, 2.16 \mathrm{~kg}\right)$. A modified montmorillonite (Closite $20 \mathrm{~A}$, abbreviated 20A, Southern Clay Products; Gonzales, TX, USA) was used. The montmorillonite was ion-exchanged with dimethyl dihydrogenated tallow ammonium ions. The tallow was predominantly composed of octadecyl chains with a smaller number of lower homologs. The approximate composition was $65 \% \mathrm{C}_{18}, 30 \% \mathrm{C}_{16}$ and 5\% $\mathrm{C}_{14}$. High-purity FS (Aldrich, Milwaukee, WI, USA) and TBPB peroxide (Aldrich) were used without further purification. LCB-PPs and several types of LCB-PP/silicate composites with varying compositions of organically modified clays were prepared by melt compounding at $180^{\circ} \mathrm{C}$ using a capillary-type compounder with a chamber size of $30 \mathrm{~cm}^{3}$. The piston was cycled at a rate of 50 per minute, and the mixing time was $10 \mathrm{~min}$ for all experiments.

\section{Measurements}

The chemical structure of purified LCB-PP was confirmed by Fourier transform infrared (FT-IR). The FT-IR spectra were recorded on a Perkin Elmer Spectrum 1000 FT-IR spectrometer (San Jose, CA, USA) with a $4-\mathrm{cm}^{-1}$ resolution. The LCB-PP was dissolved in xylene at $180^{\circ} \mathrm{C}$, cooled to $90^{\circ} \mathrm{C}$, and then stirred with chloroform for $1 \mathrm{hr}$. Following phase separation, the LCB-PP was filtered, and the residual additives were extracted with chloroform for $6 \mathrm{~h}$ using the Soxhlet method. X-ray diffraction (XRD) was carried out with a Rigaku X-ray generator (The Woodlands, TX, USA) (CuK $\alpha$ radiation with $\lambda=0.15406 \mathrm{~nm})$ at room temperature. The diffractograms were scanned in the 20 ranges from $1.2^{\circ}$ to $20^{\circ}$ at a rate of $2^{\circ} \mathrm{min}^{-1}$. A transmission electron microscope (Philips CM20, Amsterdam, Netherlands) was used to observe the dispersibility of the clay in the hybrids at an acceleration voltage of $120 \mathrm{kV}$. A 70-nm-thick, ultra-thin section was prepared using an ultramicrotome Leica EM FCS (Leica, Wetzlar, Germany). The melting points and exothermic peak temperatures were determined using a TA Q20 differential scanning calorimeter (TA Instruments, New Castle, DE, USA) with a cooling system. All samples were heated to $200{ }^{\circ} \mathrm{C}$ at a heating rate of $20^{\circ} \mathrm{C} \cdot \mathrm{min}^{-1}$, and the samples were cooled to $40^{\circ} \mathrm{C}$ at three different cooling rates. All measurements were performed under an atmosphere of dry nitrogen. The rheological properties were monitored using an oscillatory viscometer (Anton Paar, MCR 301; 8054 Graz, Osterreich, Austria) in a parallel-plate-type geometry $\left(12.5 \mathrm{~mm}\right.$ radius) at $200^{\circ} \mathrm{C}$. The gap between the plates was $1.2 \mathrm{~mm}$. XRD samples and viscometer samples were molded at $200^{\circ} \mathrm{C}$ with a laboratory hot press under approximately 10 metric tons and dried in a vacuum oven for $24 \mathrm{~h}$ before the test. The disk-shaped samples were $25 \mathrm{~mm}$ in diameter and $2 \mathrm{~mm}$ thick; the XRD samples were $1 \mathrm{~mm}$ thick.

\section{RESULTS AND DISCUSSION}

Basic properties of the LCB-PP and LCB-PP/silicate composites LCB-PP and several types of the LCB-PP/silicate composites of varying compositions were prepared by melt grafting in the presence of FS and TBPB using a micro-compounder at $190^{\circ} \mathrm{C}$. Figure 1 depicts one of the possible mechanisms for the reaction based on information available in the literature. ${ }^{3}$ Primary radicals formed by the decomposition of TBPB react with PP to yield macro-radicals. Theses macro-radicals can, in principle, undergo $\beta$-scission of the chain to form an unsaturated PP chain end and secondary radicals. The macro-radicals can simultaneously react via addition reactions with FS to yield the corresponding adduct, which can further react with the secondary macro-radicals in their vicinity to form the desired LCP-PP. In an alternative synthetic route, the adduct forms the corresponding grafted product, that is, PP-g-FS, following the abstraction of a hydrogen atom from a neighboring PP chain. The remaining double bond on the FS moiety can likewise participate in the formation of LCB-PP. The formulation and the thermal properties of the composites are summarized in Table 1.

To certify the branching reaction, the FT-IR spectra of the purified samples are shown in Figure 2. The FS grafting reaction was confirmed from the intensity of the $3100 \mathrm{~cm}^{-1}$ band that represents the $-\mathrm{C}=\mathrm{C}-\mathrm{H}$ group, and the thickness variation of each sample was corrected by the $1200-\mathrm{cm}^{-1}$ intensity for $\mathrm{C}^{-} \mathrm{CH}_{3}$ groups on the PP chains. $^{26}$

Figure 3 shows the XRD patterns of the LCB-PP/silicate composites. The interlayer distance is determined by the XRD peak using the Bragg equation as follows:

$$
2 d_{001} \sin \theta=\lambda
$$

in which $d_{001}$ is the interplanar distance of the (001) diffraction face, $\theta$ is the diffraction position and $\lambda$ is the wavelength. The X-ray patterns clearly indicate that the interlayer spacing decreases with increasing silicate content. The interlayer spacing of the silicate, PPF07-S1, PP-F07-S5, and PP-F07-S7 are 2.55, 3.37, 3.13 and $3.03 \mathrm{~nm}$, respectively.

To confirm the nanoscale dispersion of the silicate, the morphologies of the composites were observed using transmission electron microscopy. In Figure 4, the dark lines represent the silicate layers. Although some parts of PP-F07-S5 are not dispersed, each layer of clay is disordered and dispersed homogeneously in the composite, as shown in Figure 3. This finding is consistent with the weak (001) plane peak in Figure 3. However, PP-F07-S7 exhibited a slightly thick silicate layer compared with PP-F07-S5, indicating that the silicate dispersion of PP-F07-S5 is more homogeneous than that of PP-F07-S5. 


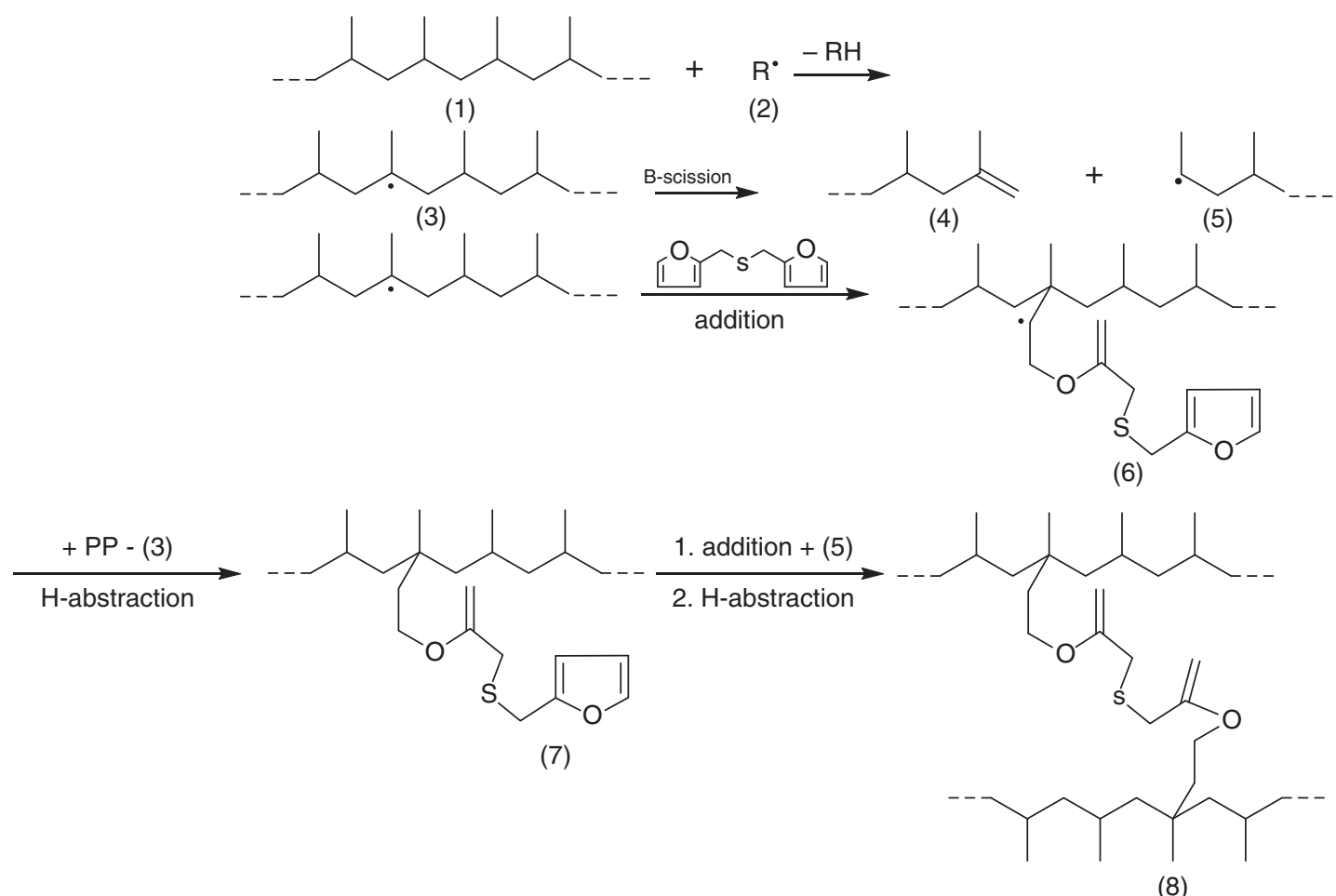

Figure 1 Schematic presentation of the reaction mechanism involving FS.

Table 1 Formulations and thermal properties of the PP and the composites

\begin{tabular}{|c|c|c|c|c|c|c|c|}
\hline \multirow[b]{2}{*}{ Sample } & \multirow[b]{2}{*}{$P P(w t \%)$} & \multirow[b]{2}{*}{$20 A(w t \%)$} & \multirow[b]{2}{*}{ FS (wt\%) } & \multirow[b]{2}{*}{$\mathrm{T}_{m}\left({ }^{\circ} \mathrm{C}\right)$} & \multicolumn{3}{|c|}{$\mathrm{T}_{p}\left({ }^{\circ} \mathrm{C}\right)$} \\
\hline & & & & & -5 & -10 & $-20^{a}$ \\
\hline PP & 100 & 0 & 0 & 164.0 & 114 & 110 & 105 \\
\hline PP-F01 & 99.9 & 0 & 0.1 & 164.3 & - & - & - \\
\hline PP-F03 & 99.5 & 0 & 0.5 & 164.7 & - & - & - \\
\hline PP-F07 & 99.3 & 0 & 0.7 & 165.2 & 122 & 111 & 113 \\
\hline PP-F07-S1 & 98.3 & 1 & 0.7 & 164.2 & - & - & - \\
\hline PP-F07-S5 & 94.3 & 5 & 0.7 & 163.6 & 120 & 112 & 113 \\
\hline PP-F07-S7 & 92.3 & 7 & 0.7 & 164.0 & & & \\
\hline
\end{tabular}

Abbreviations: FS, furfuryl sulfide; PP, polypropylene.

${ }^{\mathrm{a} C o o l i n g ~ r a t e ~}\left({ }^{\circ} \mathrm{C}\right.$ per $\left.\mathrm{min}\right)$

\section{The rheological properties of LCB-PP and the LCB-PP/silicate composites}

The rheological properties in the molten state are sensitive to changes in the molecular structure such as chain branching (or molecular weight distribution), cross-linking and the formation of a network due to physical jamming of the clay. Oscillatory measurements were used to observe the effects of the molecular structure on the rheological properties of the LCB-PP and the LCB-PP/silicate composites. The complex viscosities obtained for the LCB-PP and the silicate composites are shown in Figure 5. The measured temperature and the strain were $200{ }^{\circ} \mathrm{C}$ and $20 \%$, respectively. All data presented in this paper were verified to be linear at $200{ }^{\circ} \mathrm{C}$ and a $20 \%$ strain. The complex viscosities of PP were nearly constant at all frequency $(\omega)$ ranges, but the composite containing $5 \mathrm{wt} \%$ silicate exhibited a completely different flow pattern. The PP-F07-S5 had a

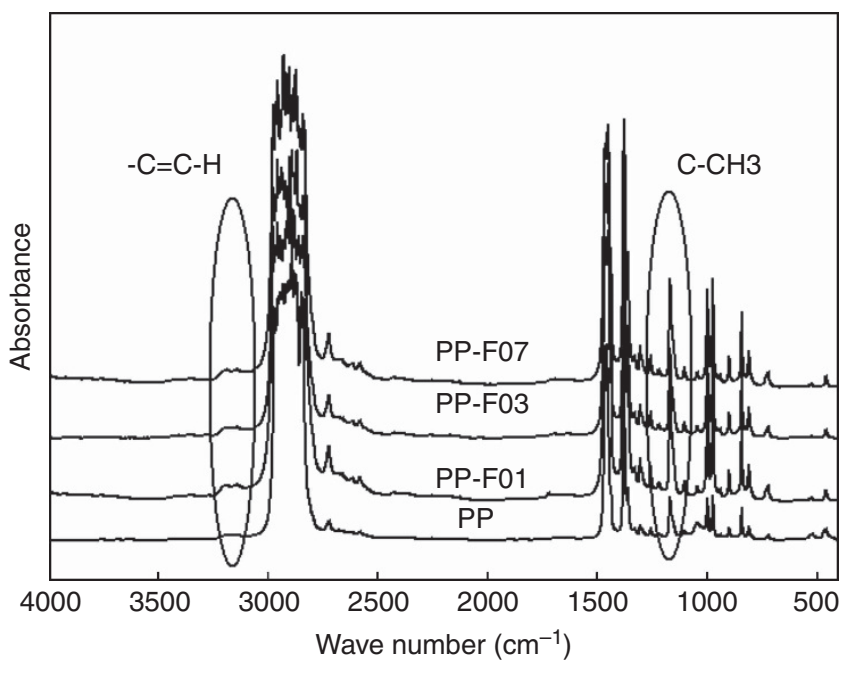

Figure 2 FT-IR spectra of the PP and LCB-PPs with varying FS content.

higher shear sensitivity and more complex viscosity at a low frequency relative to the other composites. The silicate layers in the 5-wt $\%$ silicate composite formed a more dispersed phase, as shown in Figure 4. Specifically, the flow-induced alignment of the silicates in the composite results in an increased complex viscosity and shearthinning tendency. The shear-thinning tendency was predicted by a power-low index calculated using linear regression (see Table 2).

The presence of branches can be detected by the identification and quantification of NMR signals related to the branched structure. This measurement for PP uses the resonance peaks of methine carbon atoms that correspond to branches longer than four or five Cs. ${ }^{29}$ 
However, the amount of LCB required to enhance the melt strength is too small to be detected with ordinary NMR measurements. Rheology has proven reliable for the verification of the existence of long branches on the polymer chain and is easy to implement. LCB increases the possibility for entanglements in the polymeric melt, and thus, the elasticity. Tsenoglou and Gotsis ${ }^{4}$ have shown that the introduction of sparse LCB onto PP chains at constant weightaverage molecular weight, $M_{\mathrm{w}}$, increases the zero-shear viscosity, $\eta_{0}$, of the melt but does not broaden the MWD. This increase in the $\eta_{0, \mathrm{LCB}}$ of LCB is quantitatively related to $B_{n}$ :

$$
B_{n} \approx \frac{\ln \left(\frac{\eta_{0, \mathrm{LCB}}}{\eta_{0}}\right)}{\alpha\left(\frac{M_{\mathrm{w}}}{M_{\mathrm{c}}}-1\right)-3 \ln \left(\frac{M_{\mathrm{w}}}{M_{\mathrm{c}}}\right)}
$$

$M_{\mathrm{c}}$ is the molecular weight at the onset of the entanglements (= $13640 \mathrm{~g} \mathrm{~mol}^{-1}$ for PP), and $\alpha$ is a constant and equals $15 / 8$ according to the molecular theory. The $M_{\mathrm{w}}$ for PP was calculated from the relationship between the zero-shear viscosity and the $M_{\mathrm{w}}$ of PP as follows: ${ }^{5}$

$$
\log \eta_{0}=-15.4+3.5 \log M_{\mathrm{w}}
$$



Figure 3 XRD patterns for the LCB-PP/silicate composites.
A crude approximation has been made in which $B_{n}$ was calculated using the above equations, that is, $\eta_{0.01 \mathrm{~Hz}}$ equals the zero-shear viscosity. A summary of the calculated values is provided in Table 2.

To further investigate the effect of branching and the presence of silicate on the rheological properties, the log storage modulus $\left(G^{\prime}\right)$ was plotted against the log loss modulus $\left(G^{\prime \prime}\right)$ (Han plot) for the LCB-PP and the LCB-PP/silicate composites as shown in Figure 6. The Han plot $^{30,31}$ is independent of the melt temperature and the weight-average molecular weight for monodispersed polymers. Most isotropic homogeneous polymer melts and solutions yield a slope of 2 on the Han plot, whereas heterogeneous polymeric systems, such as mesophase and block copolymers, have slopes of less than 2. The slope of block PP was 1.5, as shown in Figure 5. Yamaguchi et al. ${ }^{6}$ used a Han plot to explore the influence of PP branching on the microstructures. The slope of the Han plot decreased with the degree of PP branching. The slope decreased to 1.21 for the LCB-PP and to 1.07 for the PP-F07-S5. Note that a decrease in the slope indicates an increase in the elasticity and melt strength, suggesting that LCB renders the melt somewhat more heterogeneous and the introduction of silicate into the LCB-PP facilitates network formation due to the anisotropic nature of the silicate.

A simple technique has been described to quantify these changes in the elastic properties. $5,24,31$ The procedure involves plotting the $\tan \delta$ versus the frequency. Sugimoto et al. ${ }^{24}$ reported that the point of conversion from a liquid to a solid is defined as the point at which the moduli scale is identical to the frequency, $G^{\prime}(\omega), G^{\prime \prime}(\omega) \approx \omega^{\mathrm{n}}$ for $0<n<1$, or $G^{\prime \prime}(\omega) / G^{\prime}(\omega)=\tan (n \pi / 2)$ in which $n$ is the relaxation exponent.

The loss $\tan \delta$ is widely known to be independent of frequency for cross-linked polymers. Figure 7 indicates that $\tan \delta$ is independent of the frequency for PP, LCB-PP, and the composites, implying that the melt rheological behaviors of the LCB-PP and the composites correspond to those of a cross-linked polymer. This cross-linked character is related to the LCB and solid-like properties that result from the addition of the silicate.

\section{The crystallization behavior of the LCB-PP and LCB-PP/silicate composites}

Figure 8 shows the exothermic peaks of the PP, LCB-PP and the composite with $5 \mathrm{wt} \%$ silicate during non-isothermal crystallization. The peak temperatures of the curves are summarized in Table 1. The exothermic peak temperatures of the LCB-PP and the composite are

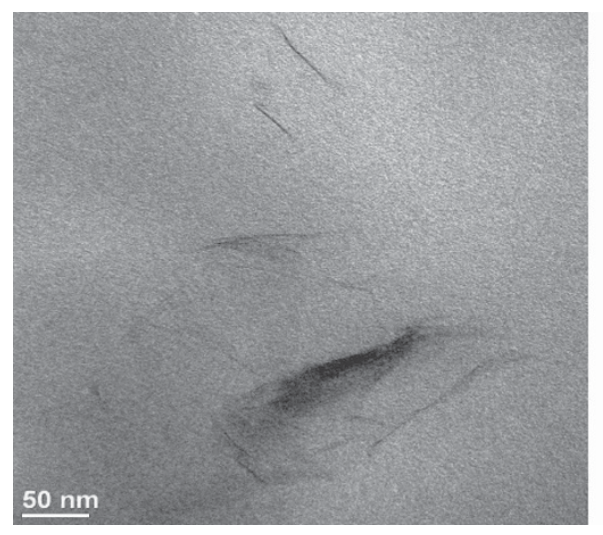

(PP-F07-S5)

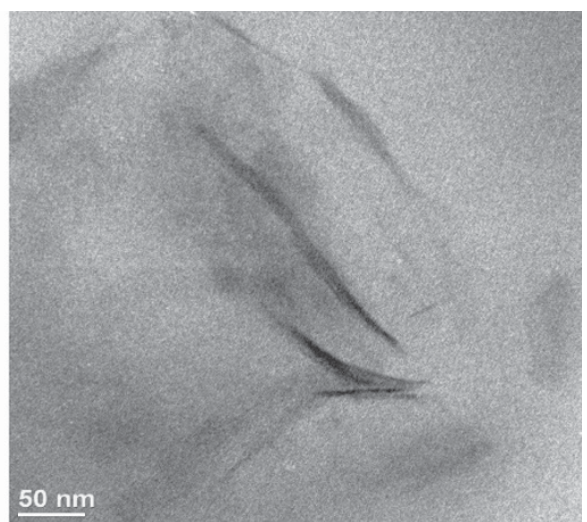

(PP-F07-S7)

Figure 4 Transmission electron microscopy images for the LCB-PP/silicate composites. 


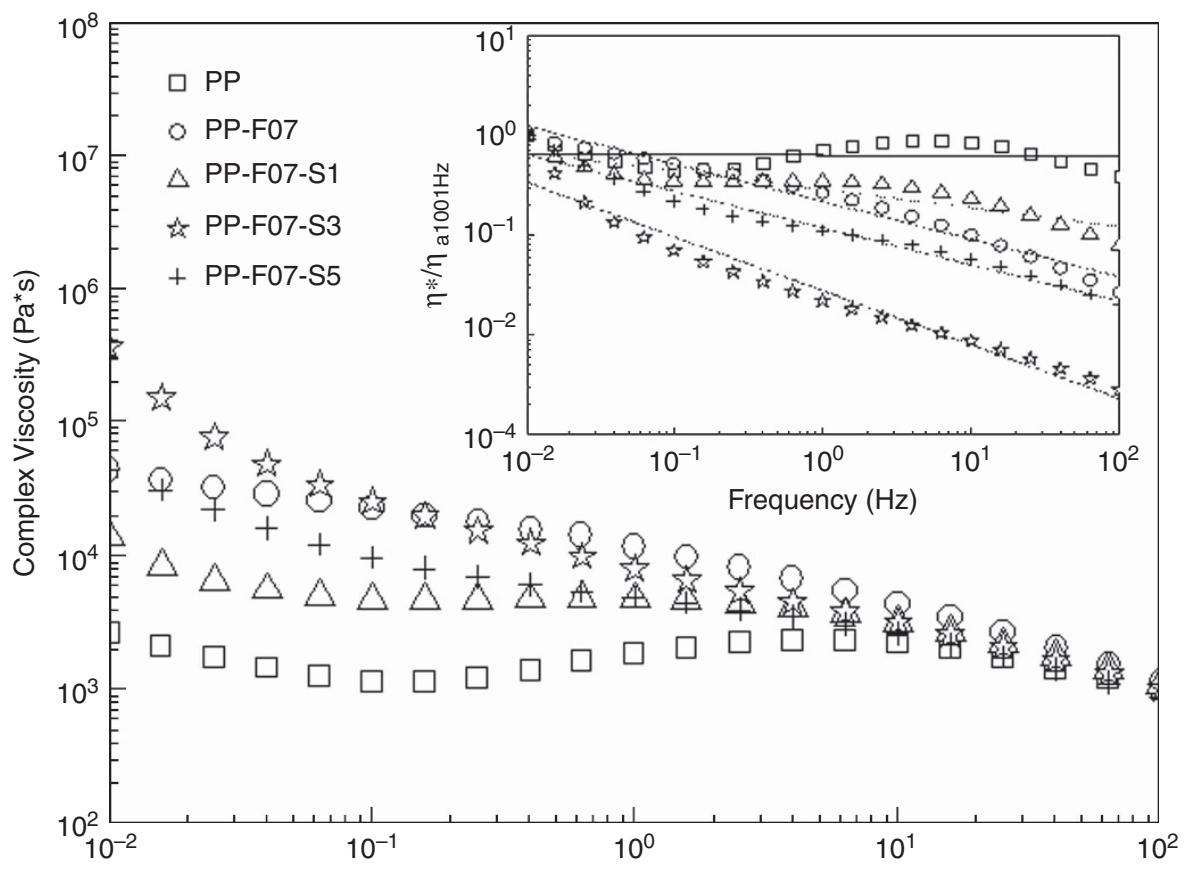

Figure 5 Complex viscosities of the PP, LCB-PP and LCB-PP/silicate composites.

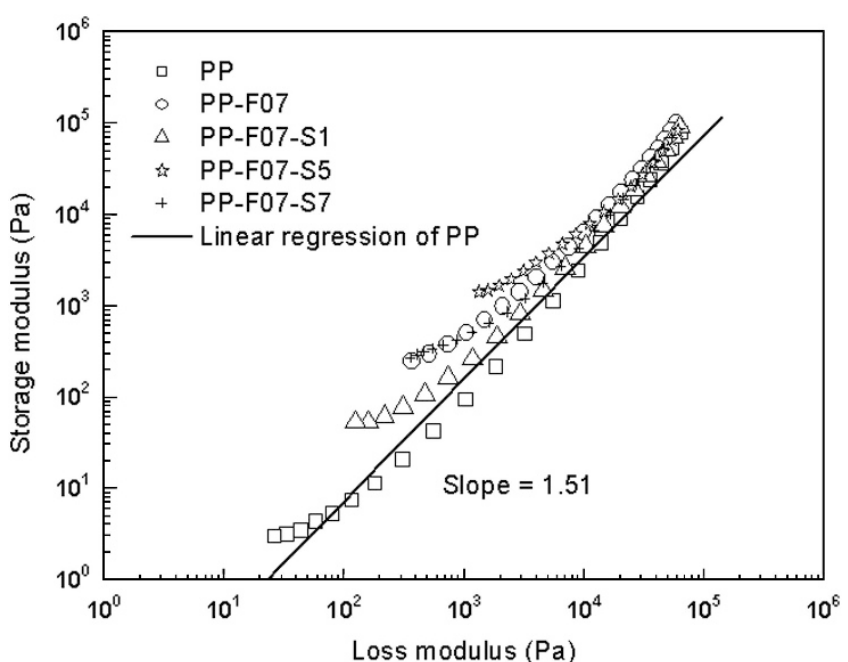

Figure 6 Plots of $\log G^{\prime}$ versus $\log G^{\prime \prime}$ for the PP, LCB-PP and LCB-PP/ silicate composites. The solid lines indicate linear regression.

Table 2 The summaries for rheological properties and weight fraction of branched chains

\begin{tabular}{lccl}
\hline Sample & $\eta_{\text {at } 0.01 \mathrm{~Hz}(\text { Pa-S })}$ & Power law index & \multicolumn{1}{c}{$B n^{\mathrm{b}}$} \\
\hline PP & 2655 & 0.99 & - \\
PP-F07 & 44550 & 0.62 & 0.10 \\
PP-F07-S1 & 14100 & 0.81 & 0.06 \\
PP-F07-S5 & 370000 & 0.46 & 0.17 \\
PP-F07-S7 & 45050 & 0.63 & 0.10 \\
\hline
\end{tabular}

Abbreviation: PP, polypropylene.

aPower law: $\eta^{*}=m(\text { Frequency })^{n}$

${ }^{\mathrm{b}} \mathrm{B} \mathrm{n}=$ average number of branches per molecule.



Figure 7 Plots of $\tan \delta$ versus frequency for the PP, LCB-PP and LCB-PP/ silicate composites.

shifted to higher values as compared with PP. In addition, the exothermic pattern of the composite is more narrow and sharp than that of PP or branched PP. Therefore, the introduction of organic clay into the PP matrix influenced the crystallization kinetics of the PP.

To analyze the non-isothermal crystallization process for the PP, LCB-PP and composite, the Avrami equation proposed by Ozawa ${ }^{20}$ was used as follows:

$$
\log [-\ln (1-C(T))]=\log \chi(T)-n \log \alpha
$$

in which $C(T)$ is the conversion at the crystallization temperature $T$; $n$ is the expanding Avrami exponent; $\chi(T)$ is the cooling function; and $\alpha$ is cooling rate. 


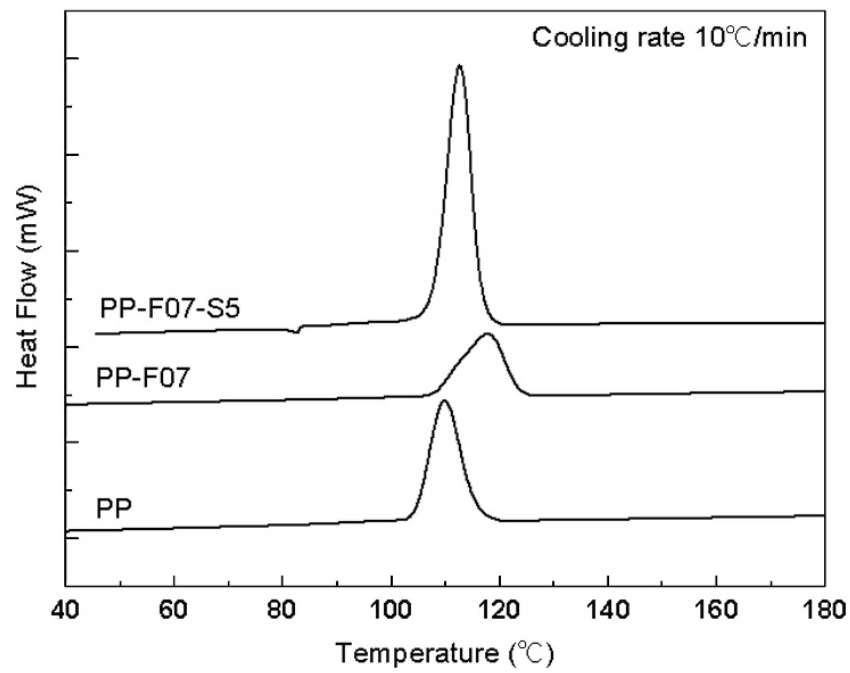

Figure 8 Cooling curves of the PP, LCB-PP and LCB-PP/silicate composites.

Figure 9 shows the conversion of the samples at $-10^{\circ} \mathrm{C}$ per min. For LCB-PP, the time approaching final crystallization increases as compared with $\mathrm{PP}$, but that for the LCP-PP/silicate composite decreases as compared with PP. The increasing time may result from an increase in the chain-folding energy of the PP chains caused by the LCB, which prevents the chains from folding back into crystal lamellas.

To further investigate the effect of modification on the crystallization kinetics, $\log [-\ln (1-C(t))]$ was plotted against $\log \alpha$ for PP as shown in Figure 10. According to the extended Avrami equation, the exponent $(n)$ can be obtained from the slope of the line, and the exponents, calculated by linear regression, are 3.6 for PP, 2.4 for LCB$\mathrm{PP}$ and 1.5 for the composite. The $n$ values close to 2,3 and 4 correspond to rod-, disk- and sphere-like growth geometries, respectively. ${ }^{20,32}$ The value of the exponent for the crystalline phase of PP is close to 4 , indicating sphere-like growth (three-dimensional growth). In contrast, the value of $n$ for the composite is close to 2 indicating rod-like growth (one-dimensional growth).

Longer and Singh ${ }^{19}$ reported that the exponent of LCB-PP was lower than that of PP. Xu and Wang ${ }^{18}$ also reported that the exponent of a $\mathrm{PP} /$ carbon nanotube (CNT) composite was lower than that of neat PP when the PP/CNT crystallization was non-isothermal.

The addition of silicates can have two effects on the crystallization behavior of $\mathrm{PP}$ in a $\mathrm{PP} /$ silicate composite. The silicates may function as heterogeneous nucleating agents for the PP crystallization, or the silicates may hinder the mobility and diffusion of the PP chains in the undercooled melt during crystallization. Decreases in the mobility and diffusion may result from an increase in the melt viscosity of the composite due to the confinement effect of the clay on the motion of the polymer chains as reported in the literature. ${ }^{9,23}$ The melt viscosity of the composite containing $5 \mathrm{wt} \%$ silicate significantly increased at low frequency as shown in Figure 5.

\section{CONCLUSIONS}

LCB-PP and several types of LCP-PP/silicate composites were prepared by melt grafting in the presence of FS and TBPB. The FS grafting level was measured from the intensity of the $3100 \mathrm{~cm}^{-1}$ band for the $-\mathrm{C}=\mathrm{C}-\mathrm{H}$ group in the FT-IR spectra. The rheological

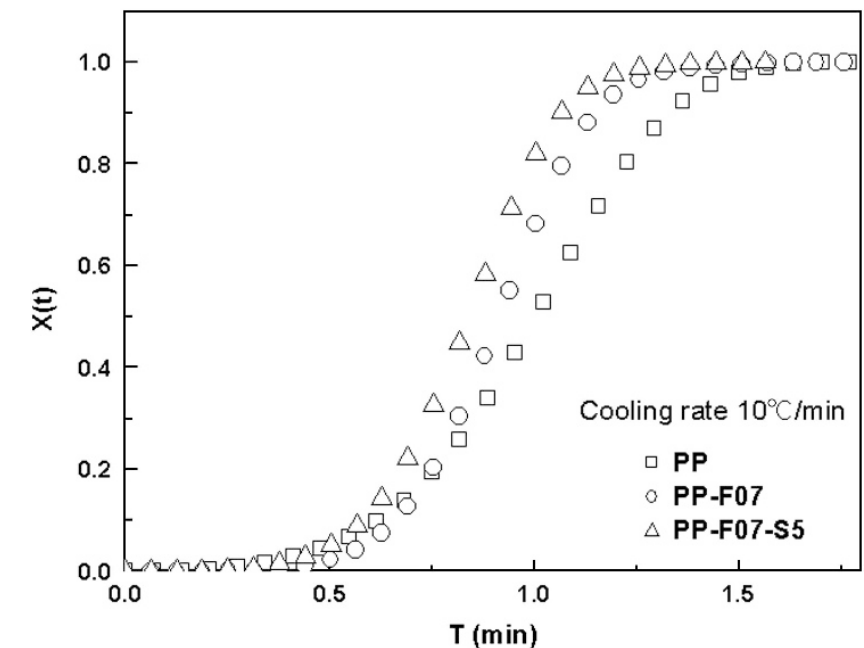

Figure 9 The conversion of the PP, LCB-PP and LCB-PP/silicate composites.



Figure 10 Plots of $\log [-\ln (1-C)]$ versus $\log \alpha$ of the PP. Solid lines indicate linear regression.

properties of the LCB-PP/silicate composites were examined by varying the silicate concentration. PP-F07-S5 showed significant increases in complex viscosity, shear sensitivity, and elastic property. The slopes of the $G^{\prime \prime}-G^{\prime}$ plots decreased to 1.21 for the LCB-PP and to 1.07 for the PP-F07-S5. This decrease in the slope indicates an increase in the elasticity and the melt strength. The enhanced elastic properties can be interpreted from the observation that the loading of silicates into PP enhances its solid-like properties in the PP melts. This result indicates that the silicates are dispersed homogeneously in the PP matrix as verified by transmission electron microscopy. The extended Avrami exponents are 3.6 for PP, 2.4 for LCB-PP and 1.5 for the composite. Values of $n$ close to 2, 3 and 4 correspond to rod-, disk- and sphere-like growth geometries, respectively.

\section{ACKNOWLEDGEMENTS}

This work was supported by Grant No. 2010-0022015 from the Basic Research Program of the National Research Foundation of Korea. 
1 Jung, H. W., Lee, J. S., Kim, W. N. \& Hyun, J. C. Study on rheological properties of polypropylene/polycarbonate blends. Korean J. Rheol. 8, 119 (1996).

2 Li, S., Xiao, M., Wei, D., Xiao, H., Hu, F. \& Zheng, A. The melt grafting preparation and rheological characterization of long chain branching polypropylene. Polymer 50, 6121 (2009).

3 Borsig, E., van Duin, M., Gotsis, A. D. \& Picchioni, F. Long chain branching on linear polypropylene by solid state reactions. Euro. Polym. J. 44, 200 (2008).

4 Tsenoglou, C. J. \& Gotsis, A. D. Logical characterization of long chain branching in a melt of evolving molecular architecture. Macromolecules 34, 4685 (2001).

5 Tian, J., Yu, W. \& Zhou, C. X. Long chain branching on linear polypropylene by solid state reactions. Polymer 47, 7962 (2006).

6 Yamaguchi, M. \& Wagner, M. H. Impact of processing history on rheological properties for branched polypropylene. Polymer 47, 3629 (2006).

7 Mousavi, S. A., Dadbin, S., Frounchi, M., Venerus, D. C. \& Medina, T. G. Comparison of rheological behavior of branched polypropylene prepared by chemical modification and electron beam irradiation under air and $\mathrm{N}_{2}$. Radiat. Phys. Chem. 79, 1088 (2010).

8 Kolodka, E., Wang, W.-J., Zhu, S. \& Hamielec, A. Long-chain branching in slurry polymerization of ethylene with zirconocene dichloride/modified methylaluminoxane. Macromolecules 35, 10062 (2002).

9 He, C., Costeux, S., Wood-Adams, P. \& John, M. Dealy, "Molecular structure of high melt strength polypropylene and its application to polymer design. Polymer 44, 7181 (2003).

10 Lagendijk, R. P., Hogt, A. H., Buijtenhuijs, A. \& Gotsis, A. D. Peroxydicarbonate modification of polypropylene and extensional flow properties. Polymer 42, 10035 (2001).

11 Choi, S. J., Yoon, K. H., Kim, H. S., Yoo, S. Y. \& Kim, Y. C. A study on the rheological properties of branched polypropylene/silicate composites. Polymer (Korea) 35, 356 (2011).

12 Li, J., Zhou, C. \& Gang, W. Study on nonisothermal crystallization of maleic anhydride grafted polypropylene/montmorillonitenanocomposite. Polym. Testing 22, 217 (2003).

13 Parent, J. S., Sengupta, S. S., Kaufman, M. \& Chaudhary, B. I. Coagent-induced transformations of polypropylene microstructure: evolution of bimodal architectures and cross-linked nano-particles. Polymer 49, 3884 (2008).

14 Istrate, O. M. \& Chen, B. Relative modulus-relative density relationships in low density polymer-clay nanocomposite foams. Soft Matter. 7, 1840 (2011).

15 Zhai, W., Park, C. B. \& Kontopoulou, M. Nanosilica addition dramatically improves the cell morphology and expansion ratio of polypropylene heterophasic copolymer foams blown in continuous extrusion. Ind. Eng. Chem. Res. 50, 7282 (2011).

16 Saujanya, C. \& Radhakrishnan, S. Structure development and crystallization behavior of PP/nanoparticulate composite. Polymer 42, 6723 (2001).

$17 \mathrm{Kim}, \mathrm{Y}$. C. Effect of maleated polyethylene on the crystallization behavior of Ildpe/clay nanocomposites. Polym. J. 38, 250 (2006).
$18 \mathrm{Xu}$, D. \& Wang, Z. Role of multi-wall carbon nanotube network in composites to crystallization of isotactic polypropylene matrix. Polymer 49, 330 (2008).

19 Lonkar, S. P. \& Singh, R. P. Isothermal crystallization and melting behavior of polypropylene/layered double hydroxide nanocomposites. Thermo. Acta 491, 63 (2009).

20 Ozawa, T. Kinetics of non-isothermal crystallization. Polymer 12, 150 (1971).

21 Lopez Manchado, M. A., Biagiotti, J., Torre, L. \& Kenny, J. M. Polypropylene crystallization in an ethylene-propylene-diene rubber matrix. J. Therm. Anal. Cal. 61, 437 (2000).

22 Lee, S. J., Kim, J. C., Cho, H. \& Kim, Y. C. Effects of maleated polyethylene on the rheological properties of LLDPE/clay nanocomposites. Polym. J. 37, 206 (2005).

23 Hyun, K., Ahn, K. H., Lee, S. J., Sugimoto, M. \& Koyama, K. Degree of branching of polypropylene measured from Fourier-transform rheology. Rheol. Acta. 46, 123 (2006).

24 Sugimoto, M., Suzuki, Y., Hyun, K., Ahn, K. H., Ushioda, T., Nishioka, A., Taniguchi, T. \& Koyama, K. Melt rheology of long-chain-branched polypropylenes. Rheol. Acta. 46, 33 (2006).

25 Yu, F., Zhang, H., Liao, R., Zheng, H., Yu, W. \& Zhou, C. Flow induced crystallization of long chain branched polypropylenes under weak shear flow. Euro. Polym. J. 45, 2110 (2009).

26 Zhang, Z., Xing, H., Qiu, J., Jiang, Z., Yu, H., Du, X., Wang, Y., Ma, L. \& Tang, T. Controlling melt reactions during preparing long chain branched polypropylene using copper N, N-dimethyldithiocarbamate. Polymer 51, 1593 (2010).

27 Qian, J., Zhang, H., Cheng, G., Huang, Z., Dang, S. \& Xu, Y. Polypropylene was (PPw)/ silica hybrid by in situ non-aqueous sol-gel process for preparation of PP/silica nanocomposites. Sol-Gel Technol. 56, 300 (2010).

28 Milner, S. T. Relating the shear-thinning curve to the molecular weight distribution in linear polymer melts. J. Rheol. 40, 303 (1996).

29 Shroff, R. N. \& Mavridis, H. Assessment of NMR and rheology for the characterization of LCB in essentially linear polyethylenes. Macromolecules 34, 7362 (2001).

30 Han, C. D., Back, D. M. \& Kim, J. G. Effect of microdomainstructure on the order disorder transition temperature of polystyrene-block-polyisoprene-block-polystyrene copolymers. Macromolecules 23, 561 (1990).

$31 \mathrm{Liu}, \mathrm{C} ., \mathrm{Li}, \mathrm{C} ., \mathrm{Chen}, \mathrm{P} ., \mathrm{He}, \mathrm{J}$. \& Fan, Q. Influence of long-chain branching on linear viscoelastic flow properties and dielectric relaxation of polycarbonates. Polymer $\mathbf{4 5}$ 2803 (2004).

32 Tabatabaei, S. H., Carreau, P. J. \& Ajji, A. Rheological and thermal properties of blends of a long-chain branched polypropyleneand different linear polypropylenes. Chem. Eng Sci. 64, 4719 (2009). 